\title{
CFD Prediction and Experimental Measurement of Blade Water Coverage in a Diesel Turbocharger
}

\author{
Jun Yao ${ }^{1}$ and Yufeng Yao ${ }^{2}$ \\ ${ }^{1}$ School of Engineering, University of Lincoln, Brayford Pool, Lincoln LN6 7TS, UK \\ ${ }^{2}$ Faculty of Science, Engineering and Computing, Kingston University, London SW15 3DW, UK
}

Correspondence should be addressed to Yufeng Yao, y.yao@kingston.ac.uk

Received 14 April 2012; Revised 3 July 2012; Accepted 19 July 2012

Academic Editor: S. Taib

Copyright ( $) 2012$ J. Yao and Y. Yao. This is an open access article distributed under the Creative Commons Attribution License, which permits unrestricted use, distribution, and reproduction in any medium, provided the original work is properly cited.

\begin{abstract}
A turbocharger unit for diesel engine is often equipped with a built-in online water washing system and its performance is not always satisfactory because of efficiency declination due to deposit accumulated on blade surfaces not being washed away. In this study, a systematic approach of using experimental measurements and computational fluid dynamics (CFD) is adopted to analyse liquid/gas two-phase flow associated with a turbocharger water washing system, in order to understand the underlying flow physics. A medium-sized diesel engine turbocharger configuration is chosen for this purpose. Experiments are focussed on blade surface temperature measurements, while CFD modelling with a coupled Eulerian/Lagrangian method is used for capturing the complex gas/liquid two-phase flow behaviours inside the induction duct and the blade passage. It was found that numerical predictions are in a good agreement with experimental data in terms of temperature distributions of the blade leading edge region and water coverage over the blade ring. Other flow features such as the water droplet trajectories and the particle size distributions are also explored and analysed in further details, and they are useful for understanding the deposit removal mechanism.
\end{abstract}

\section{Introduction}

The power output efficiency of diesel engines has been increased considerably over the last few decades, with the help of developing innovative turbocharger concept. This is particularly evident in the ship and the rail propulsion applications as well as the power generation industry [1]. A modern turbocharged engine uses the "hot" exhaust gas to spin a turbine, which drives a rotating centrifugal compressor or impeller to compress ambient air and delivers it to engine combustor. Thus, the engine power output can be increased significantly by burning more fuel. Since there is a direct link between the power output efficiency and the pressure ratio of the fuel/air mixture, the heavyduty fuel is often used that enables to achieve higher boost pressure and thus better turbocharger efficiency. However, higher contamination in this kind of low-grade fuel can cause serious corrosion and deposit accumulation that will damage the turbine blade, leading to the degradation of its aerodynamic performance. Subsequently, there is a need to assess the consequence of using heavy-duty fuel on diesel engine performance, so that, this study focuses on deposit removal technique by water washing method through experimental and computational fluid dynamics analysis of water/air two-phase flow.

The origin of deposits in a turbocharger comes from lowgrade fuel used to drive the diesel engine [2]. The engine that burns this kind of fuel most likely will be contaminated by the exhaust gas mixture from combustor with various chemical components. Deposits will be built up mainly on turbine blade surfaces and nozzle guide vanes, and consequently, the engine swallow capacity will be reduced due to the narrowing of the flow passage. In order to keep the engine performance maintained at the optimum working condition, the deposits have to be removed effectively and timely. Among various deposit removal techniques, the most cost-effective method is the "wet" cleaning, in which a water washing system is used to inject fresh water into a turbocharger from a spray ring upstream of the turbine nozzle vanes in either online and/or offline operation condition. The major risk of the online washing is that the insoluble large pieces of deposits may be washed off upstream of turbocharger blade ring 
and consequently contaminate or even impact downstream blades [3]. The offline washing offers a better solution to preserve the washing performance. However, the cost is higher due to engine shutdown. Therefore, it is desirable for a water washing system to achieve reasonable good performance under the full-loading condition without any reduction of engine unit capacity and operating speed $[4,5]$.

The performance of a water washing system depends on various factors; that is, water nozzle locations, injecting angles, injection velocity, and its interferences with mainstream gas flow. A uniform spray towards the nozzle blade ring can be achieved if water nozzle location is properly tuned such that interference between water jet flow and mainstream air flow kept to a low level [6]. A study of water washing at the full-loading or near fullloading by McDermott [7] has shown that the washing efficiency can be improved by injecting the water across the airstream, rather than align with it. It was found that the droplet size and its distributions also had significant impacts on washing efficiency, and an effective "cleaning" often requires a uniform water coverage on the full annulus of turbine blades $[8,9]$. Furthermore, the particle size and the injection speed have large influences on the impact force when the droplets impinge onto the blade surface [10]. The water spray patterns can be formed in "cone" or "flat-fan" types, depending on the liquid-gas interference and nozzle injection shape. The liquid droplet particle size distribution is dictated by the factors such as the surface tension force, the flow property in the continuous phase and the injection pressure ratio [11]. It was suggested that the droplet sizes between 150 and 250 microns could be achieved by injecting the liquid (water) at a velocity speed of $100-126 \mathrm{~m} / \mathrm{s}$ under a high operating pressure of 5-8 MPa, in order to overcome the centrifugal effect of the rotating blade rows [12]. During this process, dispersing and evaporating will happen [13]. In addition, a condensation process might be involved if the saturated vapour pressure declines with the temperature. The possible particle collision may result in new droplet-droplet interfaces, so that a secondary atomisation will take place immediately afterwards [14].

Although turbine test rig experiments are widely used for measuring surface thermodynamic properties, available test data is quite limited from public domain. This is particularly encountered for the measurement data inside the rotating passages. Therefore, computational-fluid-dynamics-(CFD-) based numerical simulations are increasingly used to study flow behaviour for representative turbocharger at a wide range of operation conditions. By using appropriate modelling methods and performing carefully designed validation processes, some complex two-phase liquid/air flow physics can be analysed at reasonable accuracy and the results can be used for validating the design, predicting the performance, and understanding the physical flow [15].

In this study, some flow characteristics in a water washing system designed for a medium sized diesel turbocharger will be studied experimentally and numerically. The flow problem is treated as gas/liquid two-phase flow type; that is, the continuous phase of the "hot" gas mixture is in singlephase form and the dispersed phase of the "cold" liquid particles are in a large number of nonuniform droplets form. The process will involve the droplets primary breakup and the secondary breakup, coupled with the liquid evaporation and condensation. The two-phase flow CFD modelling will consider flow in both the gas and the liquid phases in a coupled manner and the results will be validated against available in-house test data.

\section{Governing Equations and Computational Procedures}

The proposed two-phase flow modelling involves the Eulerian method for continuous gas phase; that is, the Reynoldsaveraged Navier-Stokes equations with turbulence models and the Lagrangian particle tracking method for water droplets within the Eulerian framework. This is widely used to simulate a dispersed particle phase in a two-phase flow for particle trajectory movement.

2.1. Eulerian Method for Continuous Gas Phase. The 3D Reynolds-averaged Navier-Stokes equations for a gas flow can be expressed as

$$
\begin{gathered}
\frac{\partial \rho}{\partial t}+\nabla \cdot(\rho U)=0 \\
\frac{\partial(\rho U)}{\partial t}+\nabla \cdot(\rho U \otimes U)=-\nabla p+\nabla \cdot \tau+S_{M}, \\
\frac{\partial\left(\rho h_{\mathrm{tot}}\right)}{\partial t}-\frac{\partial p}{\partial t}+\nabla \cdot\left(\rho U h_{\mathrm{tot}}\right)= \\
\nabla \cdot(\lambda \nabla T)+\nabla \cdot(U \cdot \tau) \\
+U \cdot S_{M}+S_{E},
\end{gathered}
$$

where $t$ is the time, $\rho$ is the density, and $U$ is the velocity vector, $p$ is the pressure. The operator $\otimes$ represents the tensor product, and $S_{M}$ is the source term, $h_{\text {tot }}$ is the specific total enthalpy and can be defined as $h_{\text {tot }}=h+(1 / 2) U^{2} \cdot \lambda$ is the thermal conductivity and $S_{E}$ is the energy source term. The term $U \cdot S_{M}$ represents the work due to external momentum source, respectively. A turbulence model is needed to provide a closure for the Reynolds stress.

2.2. Lagrangian Method for Discrete Liquid Phase. In Lagrangian particle tracking method, each droplet represents a sample of particles that follow an identical path. It is injected to achieve the "average" effect of all particles tracked, whilst the source terms for the fluid mass, momentum, and energy equations for continuous phase are calculated simultaneously [16]. The tracking procedure is applicable to a steady-state flow analysis as each particle will be tracked from an initial injection point to the final destination position at the domain exit [17]. The distinct advantage of the Lagrangian particle tracking method is due to its low computatoinal cost for flows with a wide range of particle size distributions and a complicated heat and mass transfer processes and thus, it is sufficient for industry applications.

The particle tracking is performed by a set of timedependent ordinary differential equations (ODEs) for each 
particle in terms of its space coordinates, velocity, temperature, and mass species. The velocity of water droplets is calculated at the start of the time step with initial particle velocity of $v_{p}^{o}$. The new droplet location at the end of time step is calculated by the following equation as

$$
x_{i}^{n}=x_{i}^{o}+v_{p}^{o} \times \delta t,
$$

where the superscripts " $o$ " and " $n$ " refer to old and new values, respectively, and $\delta t$ is time step.

The new particle velocity at the end of each time step is updated via the following equation:

$$
v_{p}=v_{f}+\left(v_{p}^{o}-v_{f}\right) \times e^{-\delta t / \tau}+\tau \times F_{\text {all }} \times\left(1-e^{-\delta t / \tau}\right),
$$

where $v_{p}$ is the particle velocity and $v_{f}$ is the fluid velocity, $\tau$ is the stress tensor, $F_{\text {all }}$ is the overall force acting on the particle.

2.3. Method Coupling and Submodels. The method coupling is carried out through implementation of a source term in the fluid equations. An additional transport equations for a source term as

$$
\frac{d S_{p}}{d t}=C_{S} \phi_{P}+R_{S}
$$

where $C_{S} \phi_{P}$ are the contribution from the particles that have linear variation in the solution, and $R_{S}$ includes all other contributions.

Other submodels include the buoyancy force of a particle under tracking equals to the weight difference between the fluid and the particle $F_{b}=\left(M_{P}-M_{F}\right) \times g$. The heat and mass transfer process is modelled by a liquid evaporation model. Depending on the droplet temperature of lower or higher than the liquid boiling point, it can be determined by the Antoine equation as

$$
P_{\text {vap }}=P_{\text {ref }} e^{[A-B /(T+C)]},
$$

where $A, B$, and $C$ are coefficients that are related to the physical prosperities of the material. The liquid particle is boiling if the vapour pressure $\rho_{\text {vap }}$ is greater than the gaseous pressure.

The droplets injection velocity is formulated without considering the in-nozzle cavitations as

$$
U_{P, \text { initial }}(t)=\frac{\dot{m}_{\text {nozzle }}(t)}{A_{\text {nozzle }} \rho_{P}} .
$$

The particle primary breakup is described by "Blob" model and the secondary breakup is described by Cascade Atomization, and Drop Breakup model (CAB) as

$$
\frac{r_{P, \text { Child }}}{r_{P, \text { Parent }}}=e^{-K_{\mathrm{br}}},
$$

where $K_{\mathrm{br}}$ is associated with the Weber number with $K_{\mathrm{br}}=$ $k_{1} \omega$ for $5<\mathrm{We}<80, K_{\mathrm{br}}=k_{2} \omega(\mathrm{We})^{0.5}$ for $80<\mathrm{We}<$ $350 ; K_{\mathrm{br}}=k_{3} \omega(\mathrm{We})^{0.75}$ for $350<\mathrm{We}$, respectivbely.

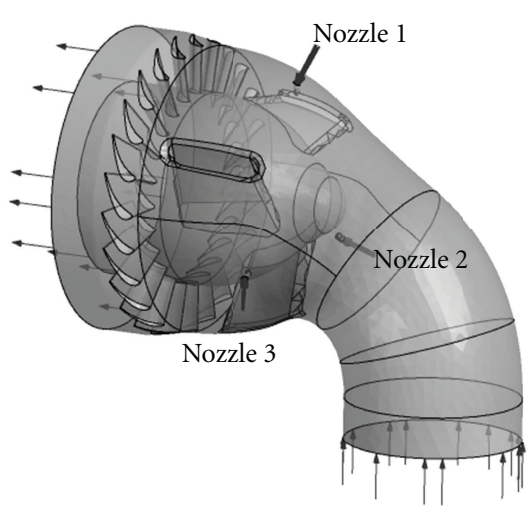

FIGURE 1: Configuration of a turbocharger with three water washing nozzles.

\section{Configurations, Flow Conditions, and Experiments}

A turbocharger configuration comprising three water injection nozzles was employed in the simulations (Figure 1). The water injectors are fitted at a short distance close to upstream of the nozzle blade ring with or without the spacer insertion. They were positioned at a nozzle injection angle of $30^{\circ}$ (denoted as standard nozzle) or $90^{\circ}$ (also denoted as spacer nozzle) against the mainstream flow direction. Experimental investigation was carried out using an inhouse test rig and numerical study of flow details, including water trajectory, droplets size, and its distributions on the blade surfaces, besides using a commercial software ANSYSCFX. Both simulation and test used same conditions; that is, the water injection pressure of 4 bars and 8 bars, and the corresponding water injection mass flow rates of $0.15 \mathrm{~kg} / \mathrm{s}$ and $0.22 \mathrm{~kg} / \mathrm{s}$, respectively. The mass flow rate at inlet is in a range of $6.0221 \mathrm{~kg} / \mathrm{s}-9.1533 \mathrm{~kg} / \mathrm{s}$. The average inflow temperature is around $520^{\circ} \mathrm{C}$. The exit pressure was tuned to meet the target of the inlet mass flow rate.

Precursor mesh convergence studies have concluded that a fine grid of 2,170,013 mesh elements is necessary to achieve the quality simulation [18]. In addition, a total of 30,000 water particles are introduced and they are injected in an uncorrelated manner into the gas flow field via three-nozzle injectors, respectively. The initial water droplet diameter was defined as $6 \mathrm{~mm}$, same as the nozzle inlet diameter. Then the primary and secondary breakup of droplets will occur immediately after the injection.

This experimental equipment is mainly used for the thermal performance measurements. The modular assembly allows the direct temperature measurements on the turbocharger nozzle guide vanes by positioning thermal couples on the turbocharger blade leading edge. The thermal couple was located on the leading edge of the nozzle guide vane of NAPIER 458 turbocharger, which comprises a total 24 nozzle blades. A total of 48 thermal couples were used with 36 thermal couples installed on the 12 blades (blade numbers 16 and blade numbers 19-24, each having 3 thermal couples) 


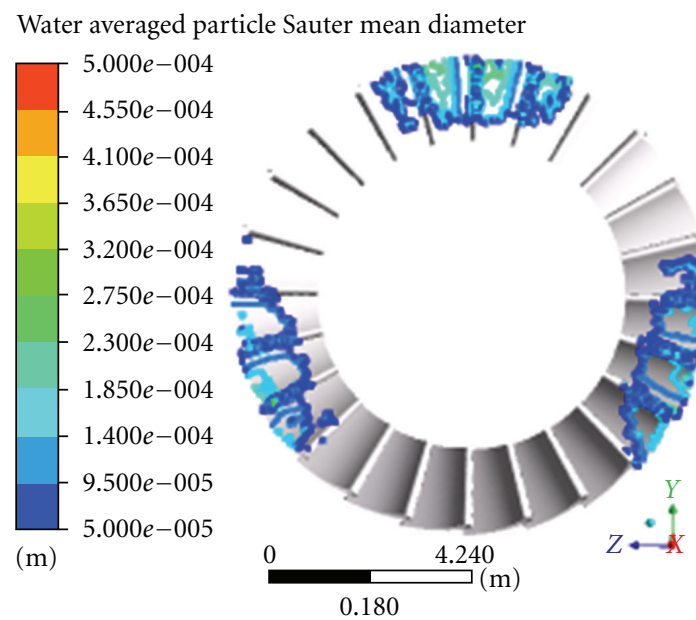

(a) 4 bars

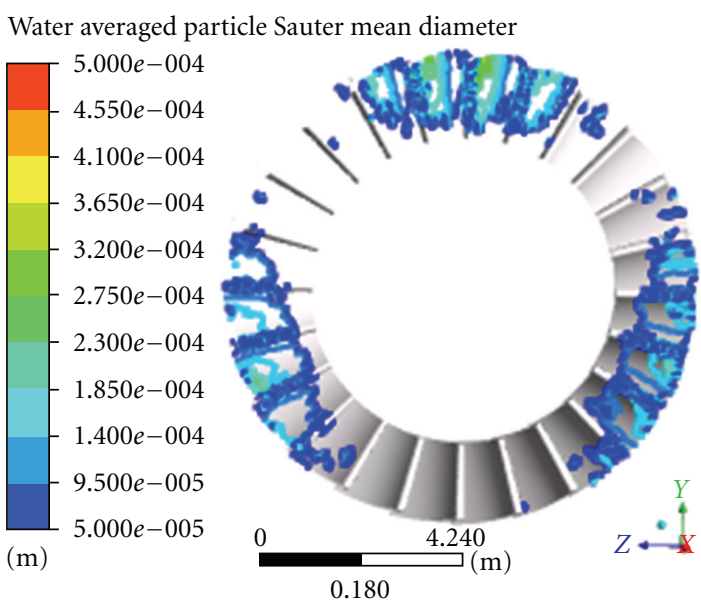

(b) 8 bars

FIgURE 2: Numerical predicted water droplet size and its distributions on the blade surface at a loading speed of $800 \mathrm{rpm} / \mathrm{T}^{\wedge} 0.5$ and $30^{\circ}$ water nozzle angle.

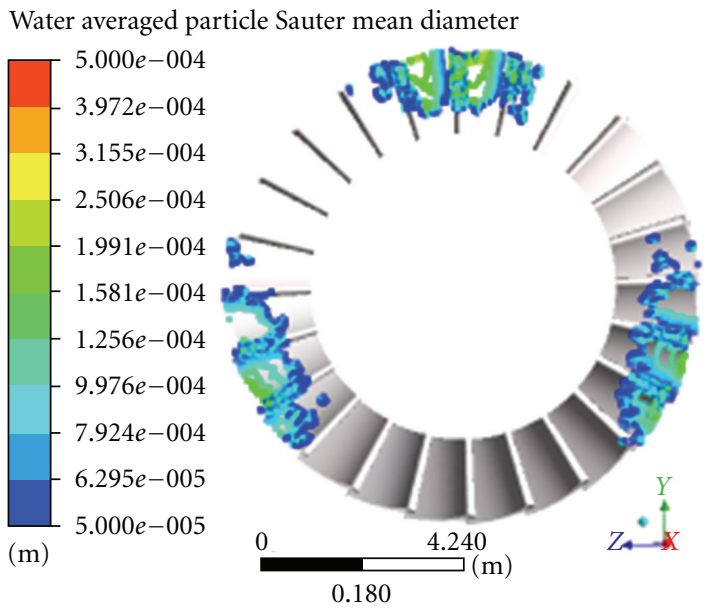

(a) 4 bars

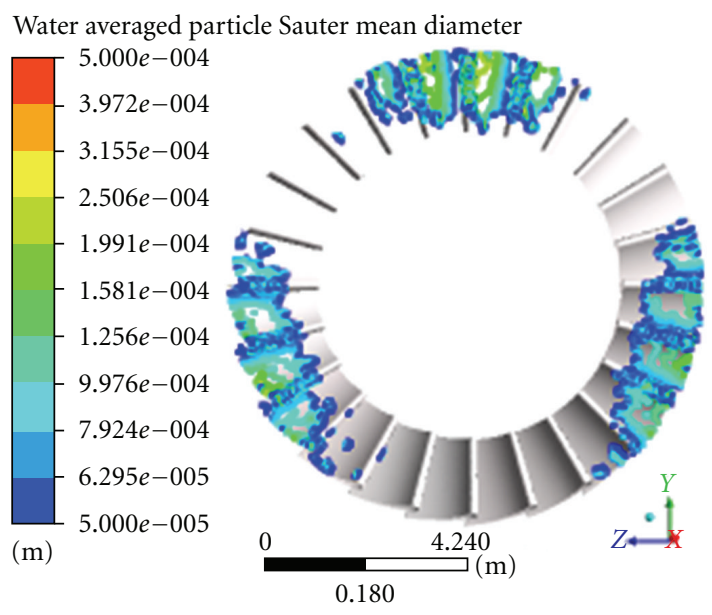

(b) 8 bars

Figure 3: Numerical predicted water droplet size and its distributions on the blade surface at a loading speed of $900 \mathrm{rpm} / \mathrm{T}^{\wedge} 0.5$ and $30^{\circ}$ water nozzle angle.

with 180 degree radius above the horizontal centreline, while 12 thermal couples installed on the rest 12 blades (blade numbers 7-18, each having 1 thermal couple). All data logging equipments used in the experimental test are from the National Instruments. The data acquisition uses the software of LabVIEW version, together with additional recording software developed at the Napier Turbochargers. Basically, the computer collects and processes the rig data and performs the overall rig operation, for instance, monitoring and performing control of turbocharger speed and "automatic" control of fuel injection, lubrication, and so forth. Details can be found in [18].

\section{Results and Discussions}

4.1. Water Injection at $30^{\circ}$ Angle. Figures 2 and 3 illustrate the water droplet coverage on the blade surface for water injection at $30^{\circ}$ angle. The droplet size in the fluid domain is predicted in a range of 50 to 500 microns for test conditions at $800 \mathrm{rpm} / \mathrm{T}^{\wedge} 0.5$ and $900 \mathrm{rpm} / \mathrm{T}^{\wedge} 0.5$, respectively. For a water injection pressure of 4 bars and the loading speed of $800 \mathrm{rpm} / \mathrm{T}^{\wedge} 0.5$, the droplet size on the blade ring is predicted between 50 and 320 microns, slightly lower than those at higher loading speed of $900 \mathrm{rpm} / \mathrm{T}^{\wedge} 0.5$. Hence, the increase of turbocharger operation speed will not cause drastic reduction of the droplet size. Similar trend is observed for test cases at a water injection pressure of 8 bars. The water droplets coverage on the nozzle blade surface is plotted to compare with the test measurement in order to validate the numerical accuracy. Figure 4 describes the numerical prediction of water droplet distributions on the nozzle blade surfaces (on the left-hand side) and the "wetted" blade water coverage determined by the experimental test (on the righthand side). The blade surface area without water droplet 


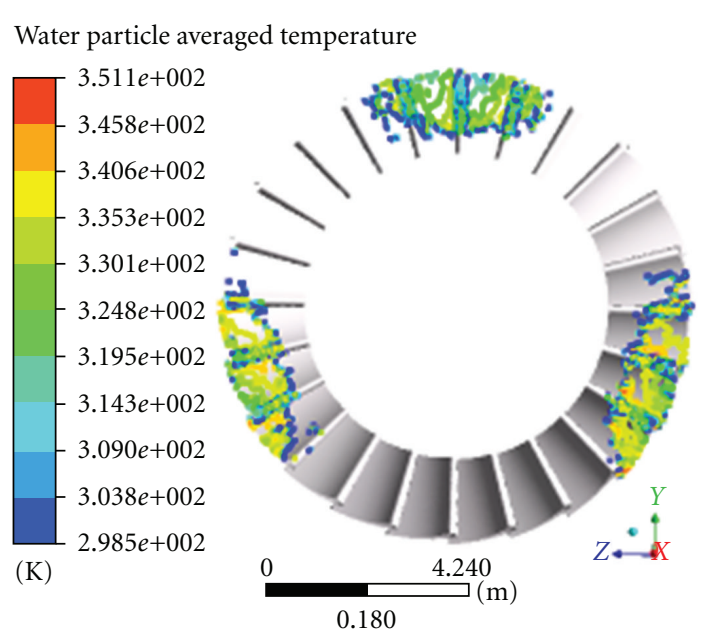

(a) $800 \mathrm{rpm} / \mathrm{T}^{\wedge} 0.5(\mathrm{CFD})$

Water particle averaged temperature

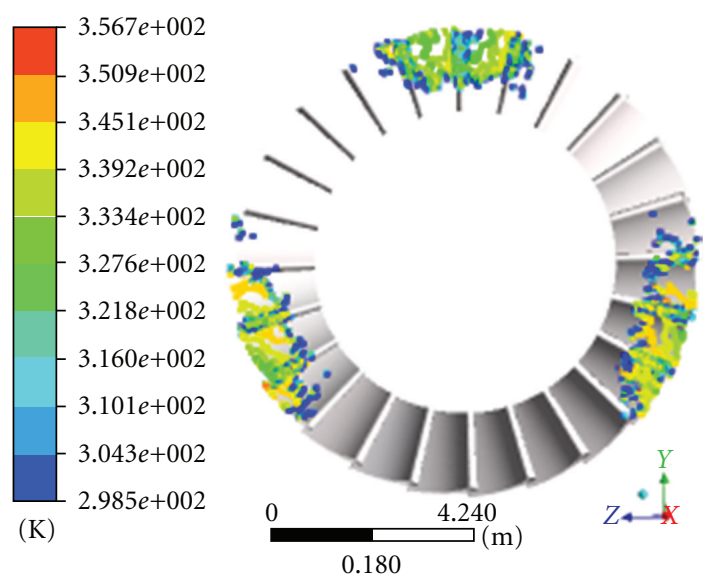

(c) $900 \mathrm{rpm} / \mathrm{T}^{\wedge} 0.5(\mathrm{CFD})$

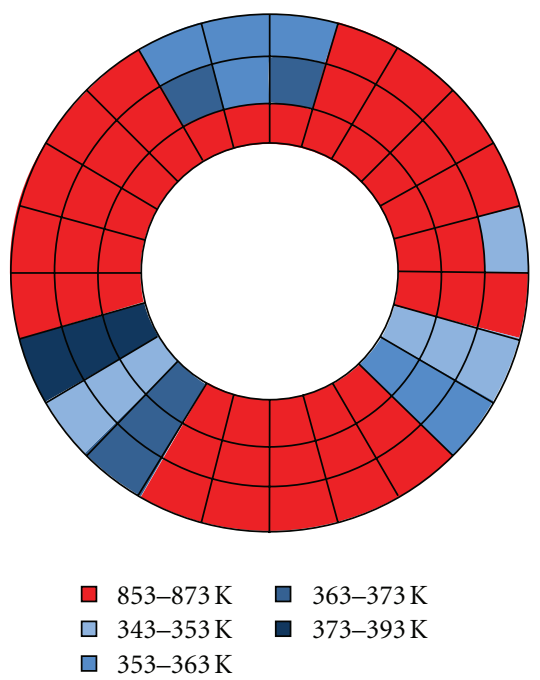

(b) $800 \mathrm{rpm} / \mathrm{T}^{\wedge} 0.5(\mathrm{EXP})$

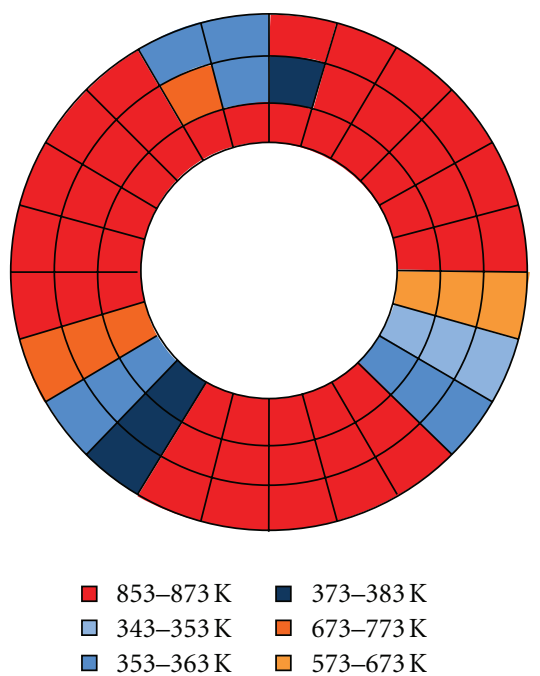

(d) $900 \mathrm{rpm} / \mathrm{T}^{\wedge} 0.5(\mathrm{EXP})$

Figure 4: Comparison of water droplet temperature variations at 4 bars on the blade surfaces between numerical predictions (CFD) and experiments (EXP), and $30^{\circ}$ water nozzle angle.

coverage is displayed by a silver colour, while those with the water coverage are indicated by a colour band, with the red colour representing the maximum droplet temperature. For a water injection pressure of 4 bars, the "wetted" surface area in terms of the blade number reduces slightly from a nondimensional speed of $800 \mathrm{rpm} / \mathrm{T}^{\wedge} 0.5$ to $900 \mathrm{rpm} / \mathrm{T}^{\wedge} 0.5$. There are two blades that have shown slightly overpredictions in terms of the droplets coverage area. For a water injection pressure of 8 bars, the water coverage has shown a similar trend (not shown here). In general, the blade surface area of water coverage decreases with the increase of turbocharger loading speed, and increases with the increase of the water washing pressure. The underprediction was observed for some blades. The predicted temperature increase of water droplet on blade surfaces is estimated at the maximum of $1.57 \%$ for a water injection pressure of 4 bars and $2.0 \%$ for a water injection pressure of 8 bars, respectively, comparing to measurement data.

4.2. Water Injection at $90^{\circ}$ Angle. Simulation of nozzle injection angle at $90^{\circ}$ angle is carried out at a loading speed of $800 \mathrm{rpm} / \mathrm{T}^{\wedge} 0.5$. Two types of nozzle installation configurations considered, one is the same as that at $30^{\circ}$ injection angle (donated as the standard nozzle) and the other was installed at the same position, but having a spacer inserted between the nozzle and the turbocharger outer casting (donated as the spacer nozzle). Both configurations were tested experimentally, and numerical results are compared with test data. For the nozzle injection with the spacer, the depth of nozzle head entering into the gas flow field is relatively shorter, while other arrangements remain unchanged. 


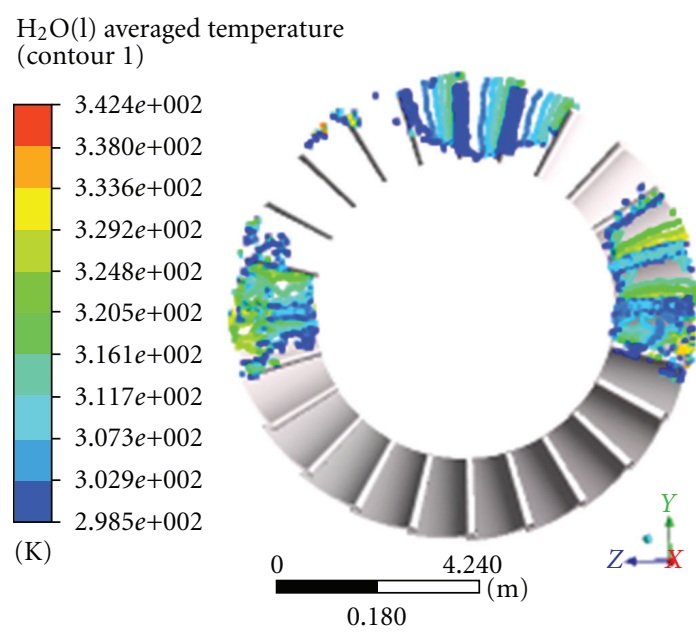

(a) 4 bars

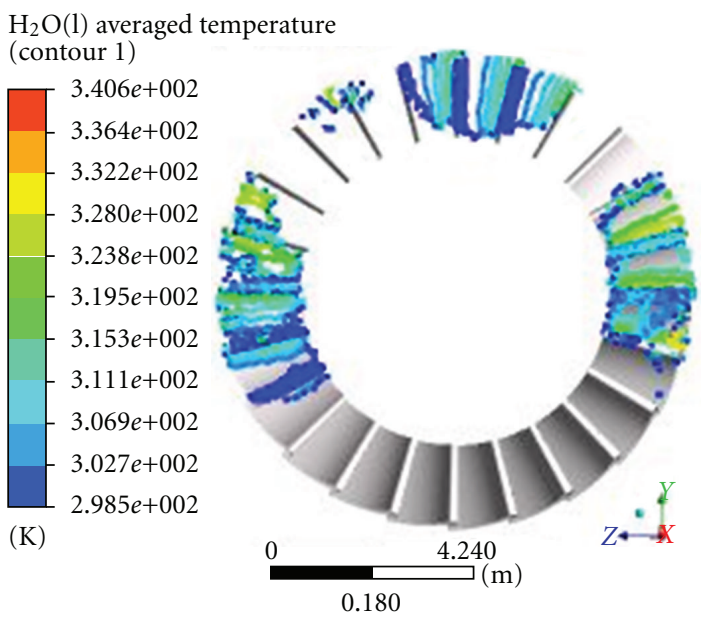

(b) 8 bars

$\mathrm{H}_{2} \mathrm{O}(\mathrm{l})$ averaged Sauter mean particle diameter (contour 1)

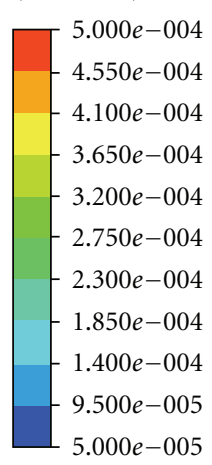

(m)

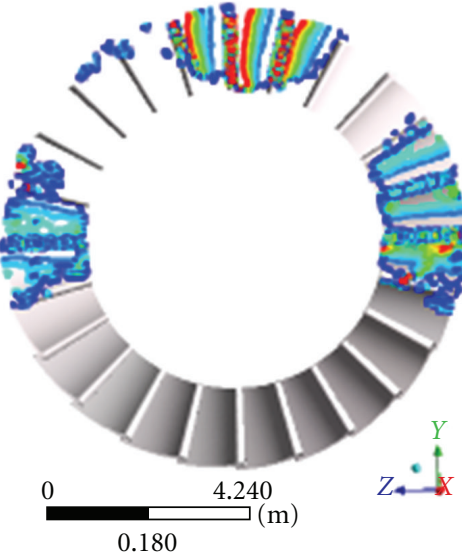

(c) 4 bars
$\mathrm{H}_{2} \mathrm{O}(\mathrm{l})$ averaged Sauter mean particle diameter (contour 1)

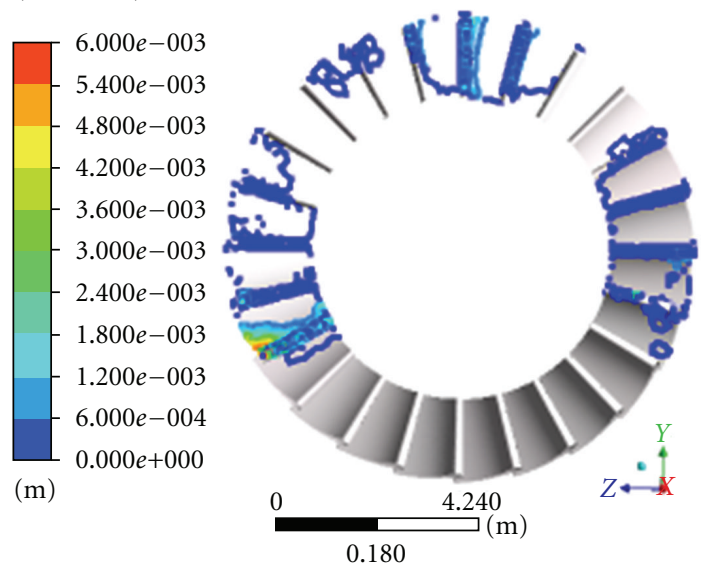

(d) 8 bars

FIGURE 5: Numerical predicted water droplet temperature and size distributions on blade surface at a loading speed of $800 \mathrm{rpm} / \mathrm{T}^{\wedge} 0.5$ for water injection pressures of 4 bars and 8 bars, respectively, and $90^{\circ}$ water nozzle angle without a spacer.

Figure 5 displays the isosurface of the averaged water droplet temperature and the size (diameter) distributions projected onto the blade surface. The trend of blade water coverage is very similar to those discussed previously for nozzle injection angle of $30^{\circ}$ and water injection pressure at 4 and 8 bars. Similarly, the number of "wetted" blades at a water injection pressure of 4 bars is lower than that at the water injection pressure of 8 bars with a high water mass flow rate. The "wetted" blades are mostly seen on the upper half of nozzle ring. The maximum temperature was found about $65^{\circ} \mathrm{C}-67^{\circ} \mathrm{C}$. The predicted droplet temperature for the water pressure at 8 bars was lower than that at 4 bars. The water droplet diameter is in a range of $5 \times 10^{-4}(\mathrm{~m})-5 \times 10^{-5}(\mathrm{~m})$, with larger droplets mostly concentrated on the upper part of the blade ring for water injection at $800 \mathrm{rpm} / \mathrm{T}^{\wedge} 0.5$.

Figure 6 gives the numerical simulations for a water injection nozzle with a spacer inserted. It shows that the number of "wetted" blades is higher than that without the spacer. The maximum temperature is found to be between $80-90^{\circ} \mathrm{C}$, which is higher compared to the standard nozzle case with $30^{\circ}$ incline angle installation. Moreover, the droplet diameters are smaller than that from the standard nozzle configuration. This may be due to the enhanced droplet evaporation and water mist at the higher temperature range. The balance of liquid evaporation rate and the correlation of droplet surface tension and temperature have also played certain roles in the dynamic process of the droplet breakup due to drag force and heat transfer effects.

For water injection at $90^{\circ}$ angle, the tendency of water droplet temperature against the diameter is fairly similar for all test cases, as described in Figures 7(a) and 7(b). It also shows that the water droplet temperature variation for the spacer nozzle is higher than that of the standard nozzle at both water pressure levels of 4 bars and 8 bars (Figure 7(b)). For the same droplet temperature, the water droplet velocity at the low water injection pressure of 4 bars is higher than that at the high water injection pressure of 8 bars (with high injection mass flow rate). The water droplet velocity from the 


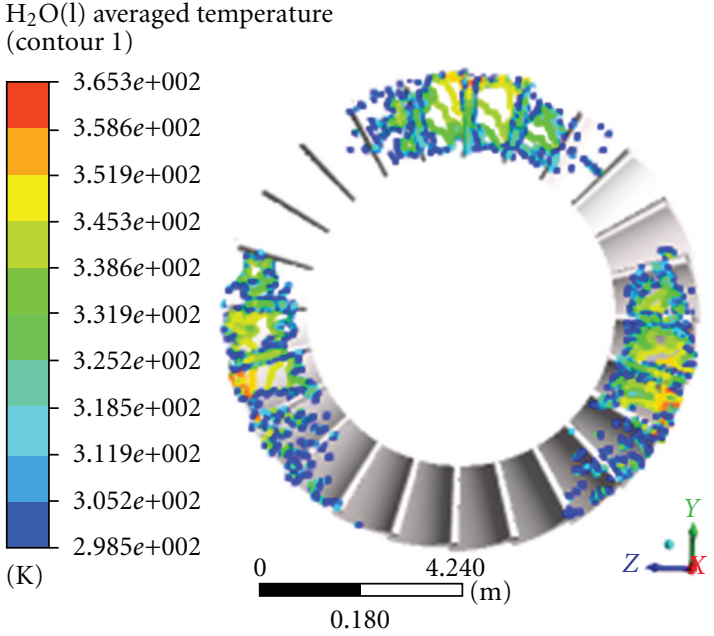

(a) 4 bars

$\mathrm{H}_{2} \mathrm{O}(\mathrm{l})$ averaged Sauter mean particle diameter (contour 1)

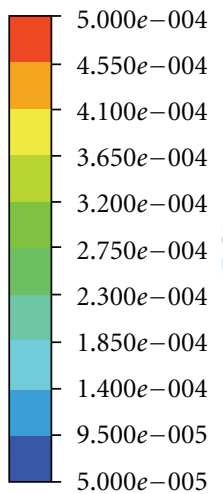

(m)

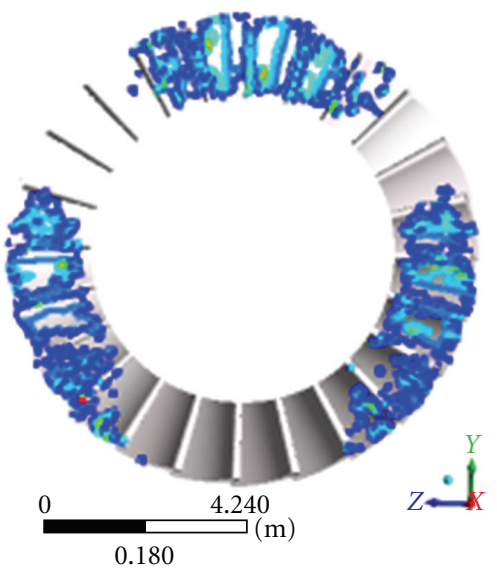

(c) 4 bars

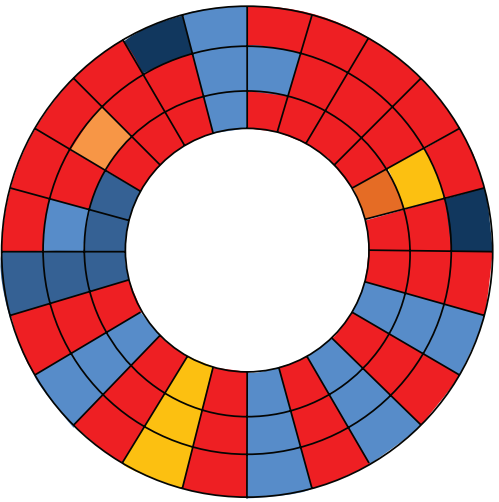

$823-873 \mathrm{~K}$

$353-363 \mathrm{~K}$

$\square 473-573 \mathrm{~K}$

(e) 4 bars (EXP
$\mathrm{H}_{2} \mathrm{O}(\mathrm{l})$ averaged temperature

(contour 1)

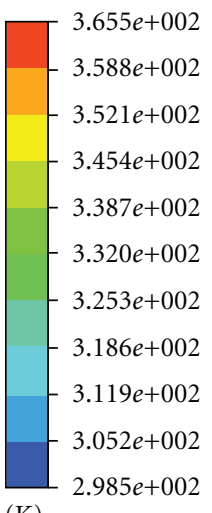

(K)

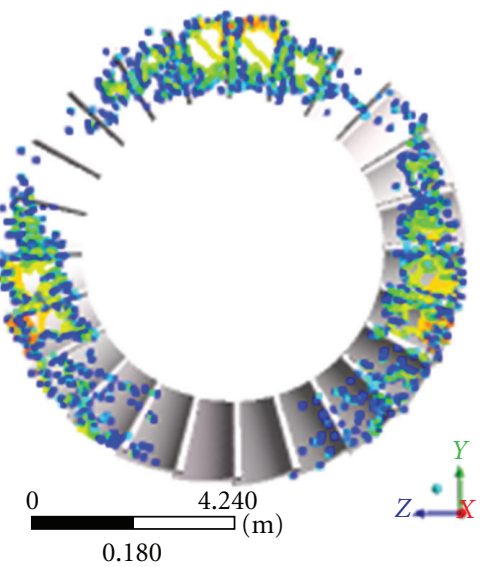

(b) 8 bars

$\mathrm{H}_{2} \mathrm{O}(\mathrm{l})$ averaged Sauter mean particle diameter (contour 1)

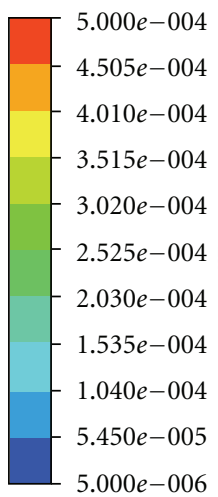

(m)

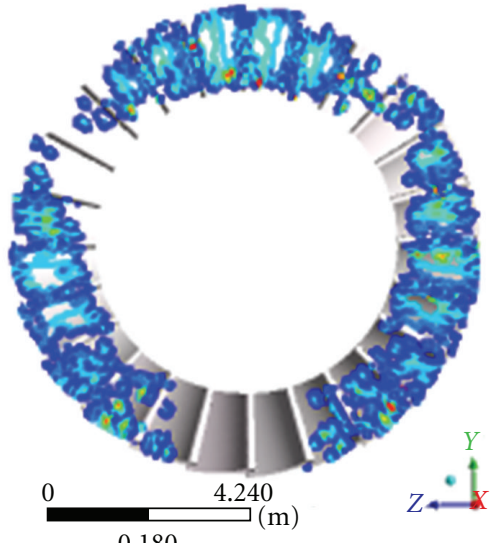

0.180 (d) 8 bars

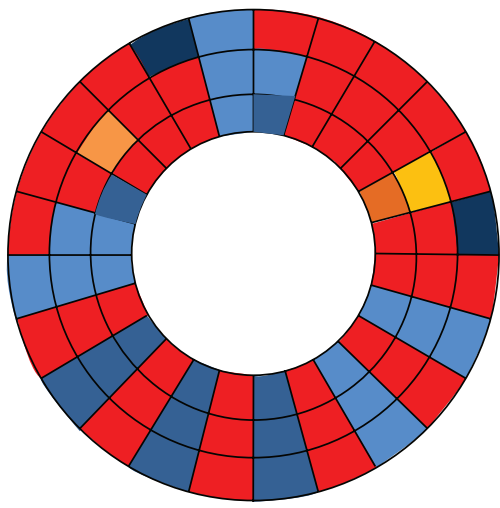

$\begin{array}{ll}\square 823-873 \mathrm{~K} & \square 373-473 \mathrm{~K} \\ \square 353-363 \mathrm{~K} & \square 573-673 \mathrm{~K} \\ \square 473-573 \mathrm{~K} & \square 673-773 \mathrm{~K}\end{array}$

(f) 8 bars (EXP)

FIGURE 6: Numerical predicted water droplet temperature and size distributions on blade surface at a loading speed of $800 \mathrm{rpm} / \mathrm{T}^{\wedge} 0.5$ for water injection pressures of 4 bars and 8 bars, respectively, and $90^{\circ}$ water nozzle angle with a spacer inserted between the nozzle and the turbocharger casting. 

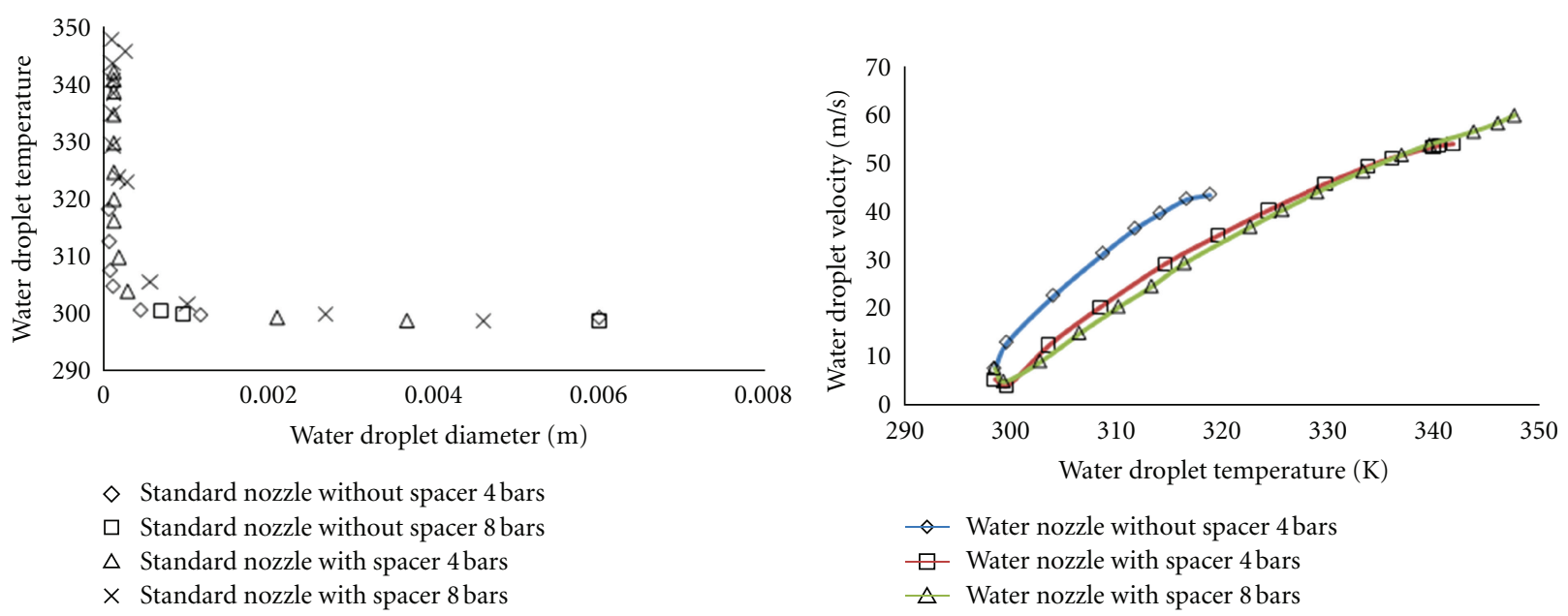

$\diamond$ Standard nozzle without spacer 4 bars

$\square$ Standard nozzle without spacer 8 bars

$\triangle$ Standard nozzle with spacer 4 bars

$\times$ Standard nozzle with spacer 8 bars

(a) Droplet temperature

(b) Droplet velocity
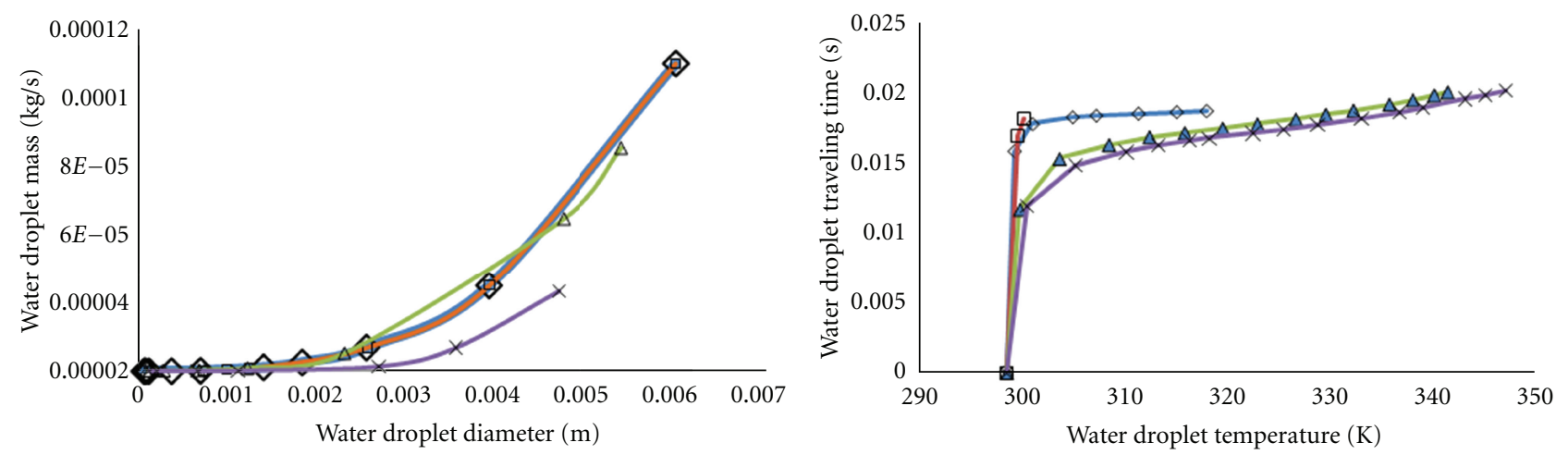

$\diamond$ Water nozzle without spacer 4 bars
$\square-$ Water nozzle without spacer 8 bars
$\Delta-$ Water nozzle with spacer 4 bars
$\star \quad$ Water nozzle with spacer 8 bars

$\diamond$ Water nozzle without spacer 4 bars

$\square$ Water nozzle without spacer 8 bars

$\triangle$ Water nozzle with spacer 4 bars

$\star$ Water nozzle with spacer 8 bars

(c) Droplet mass rate

(d) Droplet travel time

Figure 7: Comparison of water droplet behaviour at the loading speed of $800 \mathrm{rpm} / \mathrm{T}^{\wedge} 0.5$ and water injection pressures of 4 bars and 8 bars at $90^{\circ}$ water injection angle with and without a spacer.

standard nozzle is also the higher than that from the spacer nozzle. For the standard nozzle, the tendency of water droplet diameter distribution at water pressures of 4 bars and 8 bars is fairly consistent. However for the spacer nozzle at the same loading speed, the water droplet diameter at a water pressure of 4 bars is higher than that at water pressure of 8 bars. This is due to the random movement of water droplets in their trajectory direction. The comparison of experiment shows that the number of "wetted" blades using the standard nozzle agrees reasonably well with those from the experimental test at water injection pressures of 4 and 8 bars. There are no water droplet particles found on certain blade surfaces by experimental measurement, while it has been predicted by the simulation. While the experiment has shown quite random distributions of droplets on the blade ring for the spacer nozzle (see, e.g., Figures 6(e) and 6(f)), simulation does predict some regular water coverage patterns, similar to other cases. The reason for this discrepancy is not clear and probably due to either the possible measurement errors or the model limitation in the numerical simulation. Further studies in both aspects are required.

Figure 8 has shown the results of the comparison between nozzle injection angles of $30^{\circ}$ without a spacer (denoted as Geom 1), nozzle injection angles of $90^{\circ}$ without a spacer (denoted as Geom 2), and nozzle injection angles of $90^{\circ}$ with a spacer inserted (denoted as Geom 3), respectively. Generally, the tendency for all test cases is very consistent. For the standard nozzle with $30^{\circ}$ injection angle, the water droplets heating up time is the shortest at a water injection pressure of 8 bars. In contrast, a low water pressure of 4 bars has the longest water droplet heating up time (Figure 8(a)). The averaged water velocity via the water droplet temperature for the all test cases is also quite similar (Figure 8(b)). For the standard nozzles, the water droplet mass loading at a water injection angle of $30^{\circ}$ is consistent with that from a water injection angle of $90^{\circ}$. However, some variations were observed for the spacer nozzle with water injection angle of $90^{\circ}$ (Figure $8(\mathrm{c})$ ). Figure $8(\mathrm{~d})$ describes the 


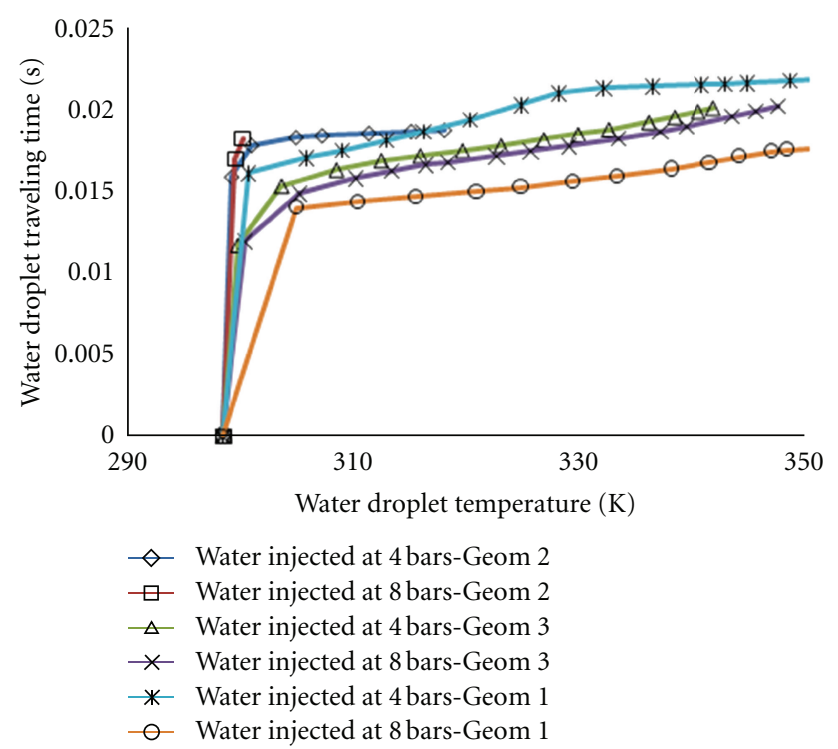

(a) Droplet travel time

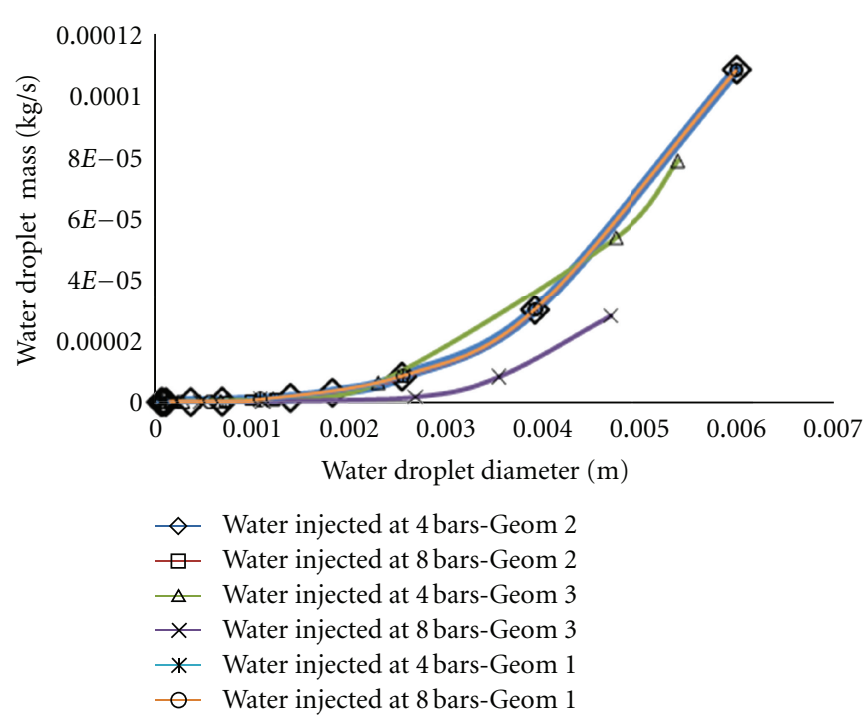

(c) Droplet mass rate

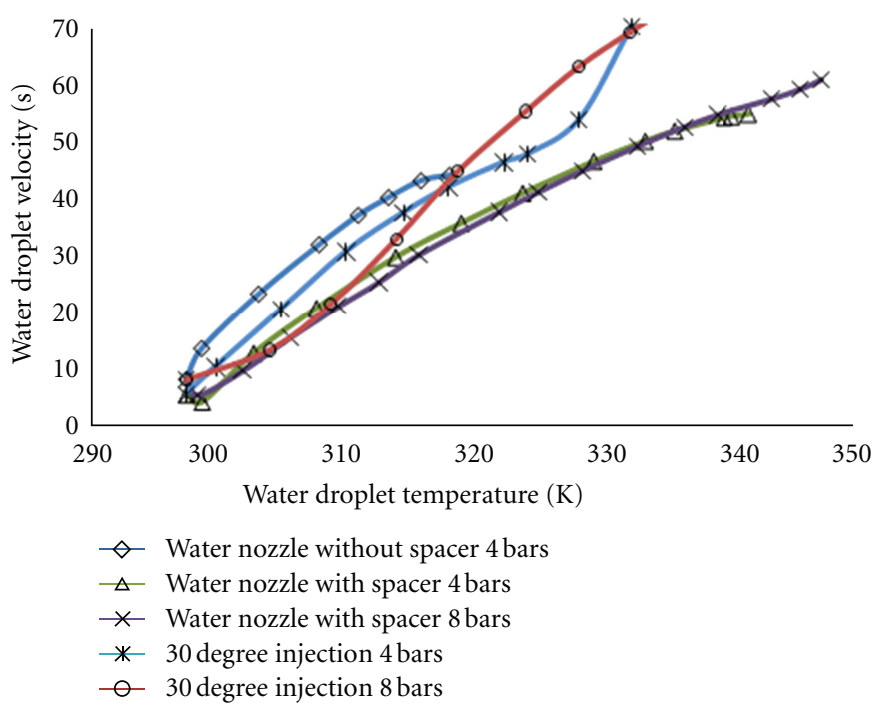

(b) Droplet velocity

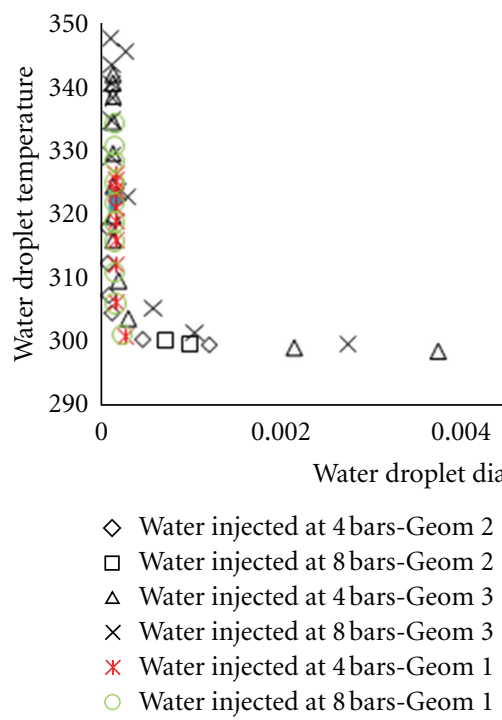

(d) Droplet temperature

FIGURE 8: Comparison of water droplet behaviour at a loading speed of $800 \mathrm{rpm} / \mathrm{T}^{\wedge} 0.5$ and water injection pressures of 4 bars and 8 bars for all three nozzles studied.

relation of the water diameter and the droplet temperature. It shows that water droplets breakup to a small size due to the primary and the secondary breakup. The water droplet temperature increases along the water droplet trajectory direction (Figure $8(\mathrm{~d})$ ).

In general, the water coverage area reduces with the increase of turbocharger loading speed. The simulation has shown that the washing performance is better at a low loading speed. However, it is not an ideal condition for industry operation as the turbocharger efficiency and power output could be significantly reduced at this lower turbocharger operational speed. Hence, it would be good to have a water washing system operated properly at a high loading speed, while maintaining desirable washing performance and efficiency.

\section{Conclusions}

Simulation has provided the water droplet details, such as the spectrum of droplet size on the blade surfaces, the temperature distribution via droplet diameter, and so forth. The pocket of water droplet trajectory is very important for an understanding of the liquid breakup process, and the modelling visualisation of droplet trajectory by isosurfaces is given to confirm the conical shape of the liquid spray. Simulation has shown that the blade water coverage for the standard nozzle water injection angle of $30^{\circ}$ is close to experimental data, especially at low water pressure level of 4 bars. Water droplet coverage at the high water pressure level of 8 bars is slightly overpredicted on some blades. For the spacer nozzle with water injection at an angle of $90^{\circ}$, 
simulation has shown the regular water coverage patterns; in contrast, the experimental measurement shows quite random distributions. The reason for this discrepancy is not clear. In this installation, the nozzle is less protruded into the mainstream gas flow. Thus, the liquid droplet and the gas flow interaction will experience considerable changes of the water coverage on blade ring, compared to the standard nozzle installation. Some model limitation in the simulation may also have some effects, for example Lagrangian method can only predict a lower volume of fraction of droplet. Thus, further investigations are needed in order to better understand the situation.

\section{Nomenclature}

$A, B, C$ : Coefficients in Antoine equation

$A_{\text {nozzle: }}$ Area of nozzle

$C_{S} \phi_{P}$ : Contribution from the particles that has linear variation in the solution

$F_{\mathrm{all}}$ : Overall forces acting on particle

$F_{b}: \quad$ Buoyancy force

g: $\quad$ Gravity accelerator

$h$ : Specific enthalpy

$h_{\text {tot }}$ : Specific total enthalpy

$K_{\text {br }}$ : Breakup constant

$k_{1}, k_{2}$ : Constants

$M_{F}: \quad$ Mass of fluid

$M_{P}: \quad$ Mass of particle

$\dot{m}_{\text {nozzle }}$ : Mass flow rate of water washing nozzle

$P_{\text {ref }}: \quad$ Reference pressure

$P_{\text {vap }}$ : Vapour pressure

$p: \quad$ Pressure

$R_{S}$ : Remaining contribution to source term

$r_{P}$ : Particle radius before breakup

$S_{E}$ : $\quad$ Source term in energy equation

$S_{M}: \quad$ Source term in momentum equation

$S_{p}: \quad$ Source term of particle

T: $\quad$ Temperature

$t: \quad$ Time

$U: \quad$ Velocity vector

$U_{P, \text { initial }}$ : Droplet injection velocity

$v_{f}: \quad$ Fluid velocity

$v_{p}: \quad$ Initial particle velocity

$x_{i}: \quad$ Initial particle location

$\rho: \quad$ Density

$\rho_{P}: \quad$ Particle density

$\rho_{\text {vap }}: \quad$ Vapour density

$\lambda: \quad$ Thermal conductivity

$\omega: \quad$ Angular frequency of particle distortion

$\delta t: \quad$ Time step

$\tau: \quad$ Shear stress tensor.

\section{Subscripts}

Child: Child particle

$i$ : Index

p: $\quad$ Particle

Parent: Parent particle.
Superscripts

$n$ : New value

$o$ : Old value.

Acronyms

CAB: Cascade atomization and breakup

CFD: Computational fluid dynamics

ODE: Ordinary differential equation

Rpm: Revolution per minute

Wb: Weber number.

\section{Acknowledgment}

The first author would like to acknowledge technical and finance support from Napier Turbocharger Ltd.

\section{References}

[1] N. Watson and M. S. Janota, Turbocharging the Internal Combustion Engine, Wiley/Macmillan, London, UK, 1982.

[2] A. Williams, Combustion of Sprays of Liquid Fuels, Elek Science, London, UK, 1976.

[3] J. P. Stalder and P. van Oosten, "Compressor washing maintains plant performance and reduces cost of energy production," in Proceedings of the International Gas Turbine and Aeroengine Congress and Exposition, ASME paper 94-CT436, June 1994.

[4] A. D. Mezheritsky and A. V. Sudarev, "Mechanism of fouling and the cleaning technique in application to flow parts of the power generation plant compressors," in Proceedings of the International Gas Turbine and Aeroengine Congress and Exposition, ASME paper 90-GT-103, June 1990.

[5] H. Margolis, "U.S. Navy on-line compressor washing of marine gas turbine engines," in Proceedings of the International Gas Turbine and Aeroengine Congress and Exposition, ASME paper 91-GT-309, June 1991.

[6] F. C. Mund and P. Pilidis, "A review of gas turbine online washing systems," in Proceedings of ASME Turbomachinery Exposition: Power for Land, Sea, and Air, ASME paper GT200453224, June 2004.

[7] P. McDermott, "Method and apparatus for cleaning a gas turbine engine," United States Patent 5, 011, 540, 1991.

[8] P. Lambart, R. Gordon, and M. Burnett, "Developments in on line gas turbine compressor cleaning," in Proceedings of the Institution of Diesel and Gas Turbine Engineers 2nd Gas Turbine Conference, pp. 136-142, Milton Keynes, UK, 2003.

[9] E. Jeffs, "Compressor washing on line for large gas turbines," Turbomachinery International, vol. 33, no. 5, pp. 49-51, 1992.

[10] J. Hayward, G. Winson, and A. Raatrae, "Wash system for gas turbine compressors," European Patent EP0933502A2, 1999.

[11] T. L. Pham and S. D. Heister, "Spray modeling using lagrangian droplet tracking in a homogeneous flow model," Automization and Sprays, vol. 12, no. 5-6, pp. 687-707, 2002.

[12] P. Asplund, "A method of washing objects, such as turbine compressors," World Intellectual Property Organization, Patent Cooperation Treaty, WO 96/40453, 1996. 
[13] M. Salewski and L. Fuchs, "Consistency issues of Lagrangian particle tracking applied to a spray jet in crossflow," International Journal of Multiphase Flow, vol. 33, no. 4, pp. 394-410, 2007.

[14] L. J. Molero de Blas, Pollutant formation and interaction in the combustion of heavy liquid fuels [Ph.D. thesis], University College, London, UK, 1998.

[15] R. S. Bunker, "A review of turbine blade tip heat transfer," Annals of the New York Academy of Sciences, vol. 934, pp. 6479, 2001.

[16] ANSYS-CFX Release version 11.0, "ANSYS-CFX-Solver Theory Guide- particle transport theory: Lagrangian Tracking Implementation," 2007.

[17] W. Holländer and S. K. Zaripov, "Hydrodynamically interacting droplets at small Reynolds numbers," International Journal of Multiphase Flow, vol. 31, no. 1, pp. 53-68, 2005.

[18] J. Yao, Numerical and Experimental Studies of Gas/Liquid Two-phase Flow in a Turbocharger [Ph.D. thesis], Faculty of Engineering, Kingston University, London, UK, 2010. 

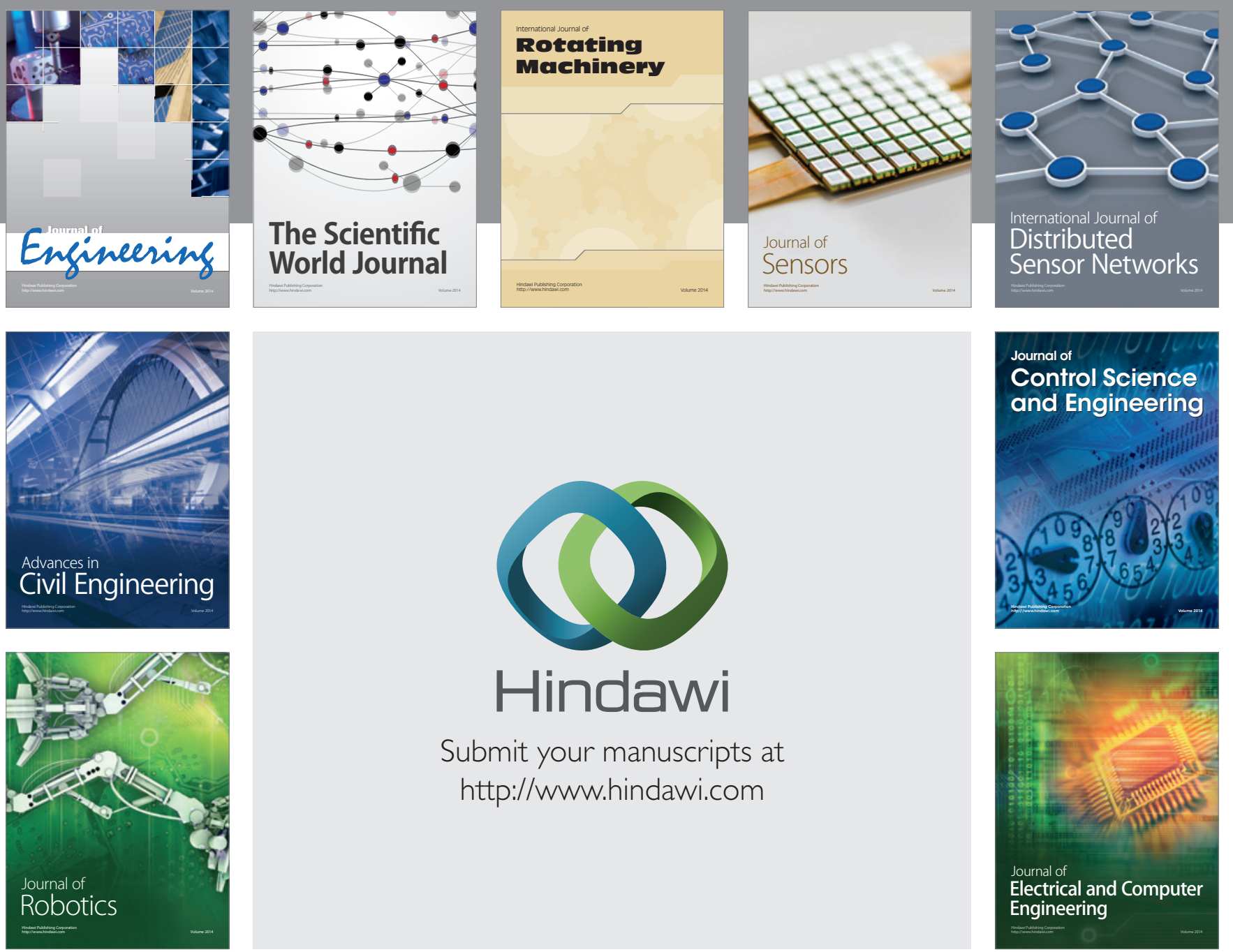

Submit your manuscripts at

http://www.hindawi.com
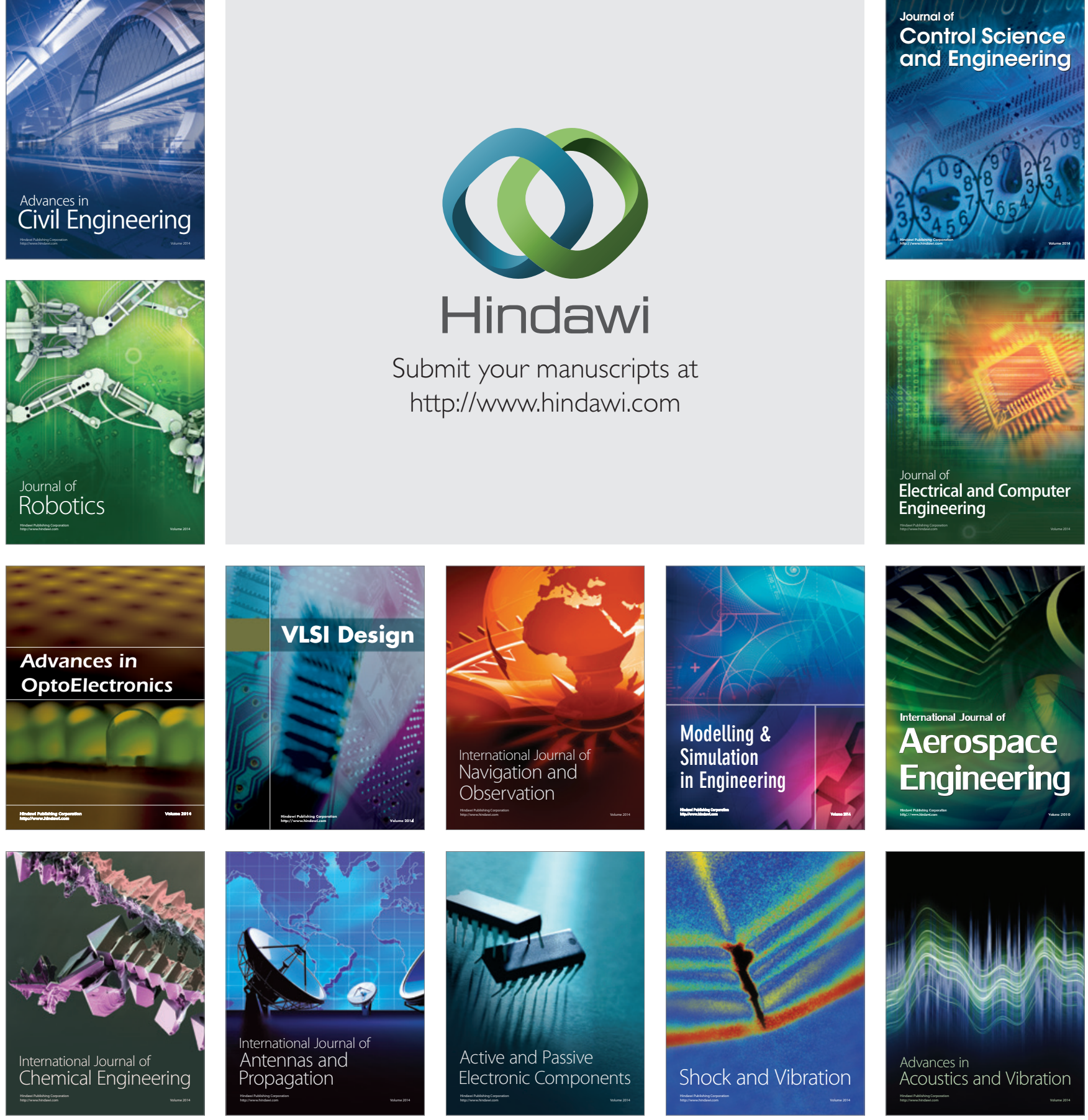